\title{
Eribulin Mesylate in Treated Metastatic Breast Cancer Patients: A Retrospective Study of Tumor Response and Adverse Effects
}

\author{
Bindu S M'1 P L Rema², Praveen Jacob Ninan ${ }^{3}$ \\ ${ }^{1}$ Assistant Professor of Radiotherapy, Govt. T D Medical College, Vandanam, Alappuzha, Kerala, India. \\ ${ }^{2}$ Professor of Radiotherapy, Govt. T D Medical College, Vandanam, Alappuzha, Kerala, India. \\ ${ }^{3}$ Associate Professor of Radiotherapy; Govt. T D Medical College, Vandanam, Alappuzha, Kerala, India.
}

Corresponding Author: Praveen Jacob Ninan

\begin{abstract}
Introduction: Breast cancer is the most common cancer, majority of patients present in advanced stage and 30\% develop distant metastasis. Metastatic Breast Cancer (MBC) is not curable and treatment aims at prolongation of life with good palliation. There is no standard treatment, though the usual first and second lines of chemotherapy include anthracyclines and taxanes. The third line chemo drugs available are gemcitabine, capecitabine, vinorelbine and eribulin mesylate

Materials and Methods: This is a retrospective study of MBC patients pretreated with anthracyclines and taxanes and then received 4 cycles of eribulin during the period March 20152017 in Medical College, Alappuzha and aims at studying the tumor response and drug toxicities. The tumor response is studied using CR-complete response, PR- partial response, PD-progressive disease and SD-stable disease.
\end{abstract}

Results: There were a total of 18 patients, majority of whom were below 50 years. ECOG performance status of $0-1$ was found in $83.3 \%$ and $77.8 \%$ were receptor positive. No patient had CR, $66.7 \%$ of patients had PR, 22.2\% had PD and $11.1 \%$ had SD. $61.1 \%$ of patients who had a PR had good performance status.55.6\% of patients who were ERPR positive had a PR and 44.4\% patients who were Her2neu positive had a PR. Most common toxicities detected were alopecia (83.3\%), neutropenia (72.2\%), fatigue (72.2\%) and neurotoxicity (55.6\%).

Conclusion: Eribulin mesylate is a drug having good response with tolerable toxicities and can be considered in our population.
Keywords: Metastatic breast cancer, eribulin mesylate, capecitabine

\section{INTRODUCTION}

Breast cancer is the most commonly diagnosed cancer and the leading cause of cancer deaths in females worldwide (1). In India the most common cancer in females is breast cancer followed by cancer of the cervix (2). The incidence of breast cancer in 2018 in India according to Globocon 2018 and NCRP (National Cancer Registry Programme) is 162,468 and it accounted for $27.7 \%$ of all newly detected cancers in women (2). In India presently $4 \%$ patients are in the 20-30 yrs age group, $16 \%$ in the $30-40$ yrs age group, and $28 \%$ in the $40-50$ age group. So almost $48 \%$ patients are below 50yrs of age (2). Regarding survival, in India the 5 year survival is less than 60 $\%$ ( 2). Majority of the patients present in advanced stage and $30 \%$ of patients presenting in early stage at diagnosis will develop distant metastatic disease(3). Even though patients with metastatic breast cancers (MBC) are not curable, survival has been significantly improved by improved options for treatment (4). The main goals of treatment in metastatic breast cancer, are prolonged survival and palliation of symptoms leading to good quality of life (5). Anthracyclines and Taxanes form the first line treatment in $\mathrm{MBC}(6)$. When patients progress with these agents second line chemotherapy with different drugs like 
capecitabine, gemcitabine, vinorelbine and eribulin are used (7). But till recently the treatment guidelines did not clearly identify a specific chemo drug or chemo schedule to be followed after the completion of the first and second line in MBC. Treatment selection depends on the tumor burden, tumor type and subtype, prior treatment exposure, and patient tolerability. Studies have shown that usage of third line and beyond third line have a potential gain in the survival and quality of life (8). Eribulin mesylate(E7389) was originally isolated from Halichondrin $\mathrm{B}$ obtained from a natural Japanese marine sponge Halichondrin okadai, but now is extracted from the synthetic analogue of Halichondrin B which was discovered by Dr Yoshito Kishi of Harvard University (9). Eribulin mesylate is approved for treatment of heavily treated MBC based on results of the break through land mark study 305 EMBRACE (9). Eribulin mesylate is a nontaxane inhibitor of microtubule dynamics and binds to the growing ends of microtubules inhibiting the microtubule growth phase without affecting the shortening phase and causing tubulin sequestration into nonproductive aggregates. The drug obtained FDA approval in November $15^{\text {th }} 2010$ for use in metastatic breast cancer. (10)

Very few studies have been conducted in Indian population regarding use of Eribulin mesylate in metastatic setting. So a retrospective data based study regarding the tumor response and adverse reactions of the drug are being studied in our patients who have received Eribulin.

\section{MATERIALS AND METHODS}

This retrospective data based study was conducted in the Department of Radiotherapy, Government Medical College, Alappuzha, Kerala. The objectives of our study are assessing the tumor response to eribulin mesylate and toxicities of the drug. After getting ethical clearance all patients with $\mathrm{MBC}$ who have already received at least two lines of chemotherapy which included both an anthracycline and taxanes, subsequently treated with eribulin mesylate for at least four cycles were selected during the period March 2015March 2017. The schedule of the treatment was eribulin $1.4 \mathrm{mg} / \mathrm{m}^{2}$ in $2-5$ minutes intravenous on days 1 and 8 , on a three weekly schedule as in EMBRACE trial(9).

\section{Inclusion criteria}

1. Patients with MBC, biopsy proven, who are pretreated with at least two lines of chemotherapy and have received both anthracyclines and taxanes and later treated with eribulin mesylate.

2. Patients should have received at least four cycles of eribulin mesylate

3. Patients had an Eastern Cooperative Oncology Group (ECOG) Performance scale of $0-2$

4. Patients with all sites of metastasis

\section{Exclusion criteria}

1. Patient with MBC whose treatment records were not available or not complete

\section{Statistical analysis}

Retrospective review of all treatment data were entered into a database for data collection and were analyzed. Data were analyzed using Microsoft excel sheet. The case registers of these patients were retrieved from our Cancer Registry and details entered into database. Responses of the tumor to the drug were assessed by way of CR-complete response, PR-partial response, PD progressive disease and SDstable disease and also recorded the adverse effects of the drug.

\section{RESULTS}

We have analyzed 18 metastatic breast cancer patients who have received four cycles of eribulin between the periods 2015-2017. 12 patients were less than 50 years of age and the youngest were 32 years old.

Performance status was assessed according to Eastern Cooperative Oncology 
Group (ECOG) scale. Fifteen patients had performance status of $0-1$ and the remaining three patients had a performance status of 2 .
Table 1 shows the ECOG grading of performance status.

Table 1: Eastern Cooperative Oncology Group (ECOG) Performance status(11)

\begin{tabular}{|c|l|}
\hline Grade & \multicolumn{1}{|c|}{ ECOG Performance status } \\
\hline 0 & Fully active, able to carry on all pre-disease performance without restriction \\
\hline 1 & $\begin{array}{l}\text { Restricted in physically strenuous activity but ambulatory and able to carry out work of a light or } \\
\text { sedentary nature eg. Light house work, office work }\end{array}$ \\
\hline 2 & $\begin{array}{l}\text { Ambulatory and capable of all self care but unable to carry out any work activities , up and about }>50 \% \\
\text { of waking hours }\end{array}$ \\
\hline 3 & Capable of only limited self care; confined to bed or chair $>50 \%$ of waking hours \\
\hline 4 & Completely disabled and cannot carry on any self care totally confined to bed \\
\hline 5 & Dead \\
\hline
\end{tabular}

Majority of patients were ERPR +ve and accounted for 14patients $(77.8 \%)$, regarding Her2neuR status 14 patients (77.8\%) were positive and 4 patients $(22.2 \%)$ were negative. All patients who were Her2neu +ve had received 12 cycles of injection trastuzumab. Majority of the patients included in the study had visceral metastasis accounting for 5 patients with liver secondaries, 6 patients with lung secondaries, 1 case of persistent axillary lymph node and 6 patients with bone secondaries. Table 2 depicts the baseline patient and tumor characteristics.

Table 2

\begin{tabular}{|l|c|c|}
\hline & Number of patients & Percentage of patients \\
\hline ECOG Performance status & & \\
\hline $0-1$ & 15 & $83.3 \%$ \\
\hline 2 & 3 & $16.7 \%$ \\
\hline Hormone status & & \\
\hline ERPR positive & 14 & $77.8 \%$ \\
\hline ERPR negative & 4 & $22.2 \%$ \\
\hline Her2neuR positive & 14 & $77.8 \%$ \\
\hline Her2neuR negative & 4 & $22.2 \%$ \\
\hline Prior chemotherapy & & \\
\hline 2-3 lines & 15 & $83.3 \%$ \\
\hline$>3$ lines & 3 & $16.7 \%$ \\
\hline Metastatic sites & & \\
\hline Liver & 5 & $27.8 \%$ \\
\hline Lung & 6 & $33.3 \%$ \\
\hline Bone & 6 & $33.3 \%$ \\
\hline Axillary node & 1 & $05.6 \%$ \\
\hline
\end{tabular}

Majority of the patients i.e. 15 patients $(83.3 \%)$ were having a good ECOG Performance Status(PS) of 0-1 and the rest $16.7 \%$ patients had a Performance Status of 2.All patients in the study have received at least two chemotherapy lines including anthracycline and taxanes. Three patients also received capecitabine after completing their second line of chemotherapy. All patients included in this study received at least four cycles of eribulin mesylate. Dose reduction was done in 3 patients following neutropenia and mucositis. Table 3 depicts the adverse effects observed in the patients

Table 3
\begin{tabular}{|l|c|c|c|}
\hline \multicolumn{1}{|c|}{ Toxicity } & Grade I & Grade II & Grade III \\
\hline Fatigue & $13(72.2 \%)$ & $5(27.8 \%)$ & 0 \\
\hline Neurotoxicity & $10(55.6 \%)$ & $6(33.3 \%)$ & $2(11.1 \%)$ \\
\hline Neutropenia & $13(72.2 \%)$ & 0 & 0 \\
\hline Thrombocytopenia & $3(16.7 \%)$ & 0 & 0 \\
\hline Anemia & $8(44.4 \%)$ & $1(05.6 \%)$ & 0 \\
\hline G I Toxicity & $8(44.4 \%)$ & $2(11.1 \%)$ & 0 \\
\hline Alopecia & $15(83.3 \%)$ & $3(16.7 \%)$ & 0 \\
\hline
\end{tabular}

Most common side effects observed in our study were neuropathy, alopecia, fatigue and neutropenia. Neuropathy may be because, all of these patients had received prior taxanes and some patients also capecitabine. Grade III neuropathy was seen only in just over $10 \%$. Fatigue may also be attributed to the additive effects of prior chemotherapeutic drugs, grade I neutropenia 
was observed in $72.2 \%$ patients. Grade II mucositis was observed in only 2 patients. In three patients dose was reduced due to neutropenia and associated mucositis, limiting the intake of the patients. Other usually observed side effects were anemia and thrombocytopenia. No patient presented with grade III neutropenia or mucositis. Table 4 depicts the tumor response.

Table 4

\begin{tabular}{|l|c|c|c|c|}
\hline \multicolumn{1}{|c|}{ Tumor Response } & CR & PR & PD & SD \\
\hline Number of patients & 0 & 12 & 4 & 2 \\
\hline Percentage of patients & $0 \%$ & $66.7 \%$ & $22.2 \%$ & $11.1 \%$ \\
\hline
\end{tabular}

CR-complete response, PR- partial response, $\mathrm{PD}$-progressive disease and SDstable disease

Response to the drug was studied with radiological investigations and clinical examination. No patient showed complete response. PR was observed in $12(66.7 \%)$ patients, disease progressed in 4 patients $(22.2 \%)$ and there was stable disease in 2 patients $(11.1 \%)$.

Tables 5 shows response in patients according to the different variables like site of metastasis, receptor status, performance status and number of chemotherapy lines already exposed to.

Tables 5
\begin{tabular}{|l|c|c|c|}
\hline \multicolumn{1}{|c}{ Tumor Response } & PR & PD & SD \\
\hline Performance status & & & \\
\hline $0-1$ & $11(61.1 \%)$ & $3(16.7 \%)$ & $1(05.6 \%)$ \\
\hline 2 & $1(05.6 \%)$ & $1(05.6 \%)$ & $1(05.6 \%)$ \\
\hline Hormone status & & & \\
\hline ERPR positive & $10(55.6 \%)$ & $4(22.2 \%)$ & 0 \\
\hline ERPR negative & $2(11.1 \%)$ & 0 & $2(11.1 \%)$ \\
\hline Her2neuR positive & $8(44.4 \%)$ & $4(22.2 \%)$ & $2(11.1 \%)$ \\
\hline Her2neuRnegative & $4(22.2 \%)$ & 0 & 0 \\
\hline Number of previous chemo schedules & & & \\
\hline $2-3$ & $10(55.6 \%)$ & $3(16.7 \%)$ & $2(11.1 \%)$ \\
\hline$>3$ & $2(11.1 \%)$ & $1(05.6 \%)$ & 0 \\
\hline Site of metastasis & & & \\
\hline Liver & $3(16.7 \%)$ & $2(11.1 \%)$ & 0 \\
\hline Lung & $4(22.2 \%)$ & $1(05.6 \%)$ & $1(05.6 \%)$ \\
\hline Bone & $4(22.2 \%)$ & $1(05.6 \%)$ & $1(05.6 \%)$ \\
\hline Axillary node & $1(05.6 \%)$ & 0 & 0 \\
\hline
\end{tabular}

When analyzing the results 11 patients $(61.1 \%)$ with PS of $0-1$ had a PR and 1 patient $(5.6 \%)$ with a PS of 2 had a PR. This shows that the tumor response is better in patients with good PS.PR was seen in 10 patients $(55.6 \%)$ exhibiting ERPR positivity and 2 patients $(11.1 \%)$ with ERPR negativity. Considering Her2neuR status 8 patients $(44.4 \%)$ who were receptor positive had a PR and 4 patients $(22.2 \%)$ were receptor negative. Analyzing patients who have received 2-3 chemo regimens, 10 patients $(55.6 \%)$ had a PR and 2 patients $(11.1 \%)$ receiving more than 3 chemo regimens received a PR. When the different metastatic sites were considered $3 / 5(60 \%)$ patients with liver metastasis had a PR, $4 / 6(66.6 \%)$ patients with lung metastasis had a PR and 4/6(66.6\%) patients with bone metastasis had a PR.

\section{DISCUSSION}

The prognosis of metastatic breast cancer continues to be poor in spite of the newer chemotherapeutic drugs. The goals of treatment of metastatic breast cancer continues to be symptom palliation which leads to better quality of life and challenge exists in selection of $\operatorname{drugs}(11)$. Improvement in overall survival is also a relevant goal of the treatment (12). There is no standard treatment protocol in MBC and selection of drug requires evaluation of patient characteristics like performance status, general condition of the patient, toxicity profile of the drug and tumor characteristics like type and subtype of tumor, receptor status, location of the metastasis and previous chemotherapy exposure. Usually single agent treatment is preferred in metastatic set-up as against multiple drugs in an adjuvant setup $(11,12)$. 
In advanced breast cancer usually first and second lines of treatment are with doxorubicin and taxanes respectively. Retrospective studies have shown that using of third line chemotherapy and beyond third line result in potential gain as each line contributes to increased survival (12). Though there is no standard third line treatment for metastatic disease the EMBRACE trial has shown a significant improvement in survival with a median overall survival (OS) of 13.1 months which was not being reported previously $(9,13)$.

In our study all patients selected were females and the youngest and oldest ages were 32 years and 62 years respectively. Majority of our patients were less than 50 years of age. In the study a total of 12 patients i.e.66.7\% obtained a partial response though none had a complete response. Progression of disease was seen in $22.2 \%$ and stable disease was observed in $11.1 \%$ of patients. This is an encouraging result though the study population is small. Majority of our patients i.e.15 out of 18 (83.3\%) had a good ECOG Performance status of $0-1$. All patients who had a PR were having a performance score of $0-1$ that is $11 / 18$ patients $(61.11 \%)$. This is in line with the major studies in this regard and performance status is considered to be a prognostic indicator.

Hormone positive patients formed the majority i.e.14/18 (77.8\%) were both ERPR and Her2neu positive. All patients with Her2neu positivity received 12 cycles of Injection Trastuzumab by properly monitoring with echo at commencement and after every three cycles. When considering ERPR status $55.5 \%$ of patients with ERPR positivity had a PR while only $11.1 \%$ patients who were ERPR negative had a PR. When considering Her2neuR status $44.4 \%$ of patients who were Her2neu positive had a PR. Disease progressed in spite of treatment in $22.2 \%$ of patients with ERPR positivity. In Her2neu positive patients $22.2 \%$ patients had progression of disease and $11.1 \%$ had a stable disease. All Her2neu negative patients had a partial response and none had progressive disease or stable disease. This is also in line with the standard trials where better response was seen with Her2neu negative receptor status. Since the study population in this present analysis is small, drug efficacy by tumor subtypes cannot be clearly identified.

Considering the number of chemo regimens received prior to eribulin mesylate $15 / 18$ i.e. $83.3 \%$ patients received only 2 chemotherapy regimens before starting eribulin which included doxorubicin and taxanes and $16.7 \%$ of patients also received third line with capecitabine. It was seen that $55.5 \%$ patients who received two lines of therapy had a PR, 16.6\% patients had a progressive disease and $11.1 \%$ patients had a stable disease. When response was looked into in patients receiving more than 2 prior chemoregimens out of three patients two had a partial response and one had a progressive disease. Since the sample size is small no definite conclusion is drawn from this.

Metastatic sites involved were bone, liver, lungs and a solitary lymph node. All patients with bone secondaries had lesion in the vertebrae and all these patients received palliative radiation to spine and were wearing spinal brace. Patient with solitary axillary node underwent excision twice and irradiated. She was kept on oral capecitabine after taking doxorubicin and taxanes. The axillary lesion was progressing in spite of all these treatment modalities. Just after 1 cycle of eribulin itself her lesion started improving and she received an excellent quality of life thereafter. She received a partial response of the tumor. Out of 6 patients with bone metastasis, 4 patients obtained a PR. In the case of lung metastasis out of 6 patients 4 patients had a PR one patient had a progressive disease and one patient had a stable disease.

Eribulin mesylate was found to have a good toxicity profile. The frequent toxicities observed were fatigue and neurotoxicity. Even though most of the patients complained of fatigue, grade III fatigue was not observed in any patients. In 
the EMBRACE study fatigue is the most common side effect observed. Chemotherapy was delayed in 2 patients who had grade II fatigue associated with neutropenia and mucositis. Neurotoxicity was the other major complaint of the patients, which may be partly because, all of these patients had already received taxane and a few had received additional capecitabine. Grade I alopecia was seen in $83.3 \%$ patients, all these patients were in the process of starting to regrow their hair after stopping treatment with doxorubicin and taxane. Grade I neutropenia was seen in $72.2 \%$ of the patients, thrombocytopenia and anemia were observed in $16.7 \%$ and $44.4 \%$ patients respectively. Patients who developed mucositis was $44.4 \%$ and had diarrhea and minimal vomiting which could be managed with symptomatics and supportives. The toxicity profile of eribulin was found to be favorable and tolerable. When the clinical response was weighed against the toxicity of the drug the conclusion reached is that eribulin should be definitely tried in indicated patients. In our study we have obtained favorable results regarding the response rate, clinical benefits and overall survival with tolerable toxicity profile. Adverse effects observed in our study did not significantly differ from that of EMBRACE study (9). The results of the Belgian expanded access programme of eribulin in the treatment of 154 pretreated metastatic breast cancer patients showed that the safety profile was predictable and the most common reported toxicities were fatigue, neurotoxicity, alopecia and neutropenia and incidence of grade III and IV neutropenia was less (14), this is in accordance with our study. In our study of limited number of patients we have observed drug activity in all subgroups of patients and all metastatic sites.

The other drugs frequently used are third line treatments are capecitabine, gemcitabine, ixabepilone, nab-paclitaxel and vinorelbine; none of these drugs is considered as standard of care (15). Capecitabine is one of the most common chemo drug used as second or third line after patient fails with anthracyclines and taxanes. Capecitabine is a drug with tolerable toxicity profile except for peripheral neuropathy and hand-foot syndrome (16). A phase III randomized trial comparing capecitabine with eribulin as second and third line has shown in its preliminary results that there is a nonsignificant improval in overall survival (HR 0.88, P 0.056) in favor of eribulin (17). Gemcitabine is a common chemodrug used as third line treatment with an overall response rate of 40-69 \% and overall survival (OS) of approximately12 months (18). When used in combination with paclitaxel there is a substantial increase in intracellular levels of active component of gemcitabine(18) Ixabepilone is a semi synthetic lactam analogue of epothilone B used as third line chemodrug in MBC and is used as monotherapy and or in combination with capecitabine (19). Vinorelbine is a vinca alkaloid which has shown response rates of $10-35 \%$ in pretreated MBC patients and can be used in combination with trastuzumab with encouraging results (20).

EMBRACE is the first and only single agent study demonstrating a significant overall survival benefit in patients with late stage metastatic breast cancer patients (9).TROTTER trial which was recently published by Garrone et al, included 113 patients from 10 Italian hospitals shown that eribulin was well tolerated and that an overall response rate (ORR) of $24 \%$ was seen in patients (21).

Considering our study the main drawback is that it is a retrospective analysis and therefore lacks a predefined study protocol. Another disadvantage of this study is its small sample size. Very few studies have been conducted in South Indian population so this gives us an idea regarding how our patients respond to this drug and the adverse effects experienced by our patients. 


\section{CONCLUSION}

The recent approval of eribulin in the treatment of metastatic breast cancers as third line has expanded the treatment options and has resulted in improving survival and quality of life in MBC patients. In our retrospective analysis we have got encouraging results with response of the tumor comparable to the standard trials, with a good toxicity profile. At present, the high cost of the drug is the limiting factor which is preventing the patients from benefitting the drug.

\section{Acknowledgement: None}

Conflicts of Interest: Authors have declared no conflicts of interest.

\section{Source of Funding: None}

\section{Ethical Approval: Approved}

\section{REFERENCES}

1. DeSantis C, Ma J, Bryan L, Jemal A: Breast Cancer Statistics CA Cancer J Clin 2014: 64: 52-62

2. Latest Statistics of Breast Cancer in India 2020: Breast Cancer India: 12;964-9 breastcancerindia.net/statistics/trends.html

3. Early Breast Cancer Trialists Collaborative Group: Effects of Chemotherapy and Hormone Therapy for Early Breast Cancer on recurrence and 15 year survival: An Overview of the Randomized trials: Lancet 2005; 365:1687-717

4. Chaisk, Speers C H, D'Yachkova Y, Kang A, Malfair- Taylor, Barnett $J$ et al. The impact of view chemotherapeutic and hormonal agents on Survival in a population based Cohort of women with Metastatic Breast Cancer 2007: 10;973-9

5. Massino Cristofanilli, G Morris Darrance Jr; Advancements in the treatment of Metastatic Breast Cancer (MBC); The Role of Ixabepilone; Journal of Oncology 2012 Feb 14(6):243-7. Article ID 703858

6. R Parideans, L Bigenzol, P Bruntg, J G Klinjin et al Paclitaxel versus Doxorubicin as first line single agent chemotherapy for Metastatic Breast Cancer a European Organization for Research and Treatment of Cancer Randomized study with crossover; J Clin Oncol 2000 Feb 18(4): 724-33

7. Gourmelon C, Frenel J S, Campone M; Eribulin Mesylate for the treatment of late stage breast cancer; Expert Opin Pharmacotherapy 2011; 12-1883-90

8. Vincenzo Adamo, Giuseppina Rosario, Rita Ricciardi, Dario Giuffrida et al Eribulin use as third line therapy in patients with Metastatic Breast Cancer; (VESPRY): A Prospective Multicenter Observational Study; Therapeutic Advances in Medical Oncology Dec 19, 2019;234:425-9

9. Cortex J, O'Shaughnessy J, Loesch D, Blum J F, Validot L T, Petrakovak et al ; Eribulin monotherapy versus treatment of Physician's choice in patients with Metastatic Breast Cancer( EMBRACE); A phase III open labeled Randomized study ; LANCET 2011; 377;914-23

10. Domoghue M, Lemery S J, Yuan W, He K, Sridhara R, et al Eribulin mesylate for the treatment of patients with refractory metastatic breast cancer:" Use of a physician's choice control arm in randomized approval trial; Clinical Cancer Research 2012;18 1496-505

11. Azam F, Latif M F et al Performance Status assessment by using ECOG(Eastern Cooperative Oncology Group) Score for cancer patients by Oncology Health Care Professionals ;Case Rep Oncol 2019;12:728-736.

12. Planchat E, Abrial C, Thivat E, et al. Late lines of treatment benefits survival in metastatic breast cancer in current practice. Breast. 2011; 20: 574-8

13. Gradishar WJ. The place for eribulin in the treatment of metastatic breast cancer. Curr Oncol Rep. 2011; 13: 11-6

14. Aftimos P, Polastro L, Ameye L et al. Results of the Belgian expanded access programme of eribulin in the treatment of metastatic breast cancer closely mirror those of the pivotal phase III trial, Cancer 2016; 60:117-24

15. Ayoub JP, Verma S, Advances in the management of metastatic breast cancer options beyond first line chemotherapy. Curr Oncol 2012, 19; 91-105

16. Kathy D miller, Linnea I, Chap, Frankie A, Holmes et al; Randomized phase trial of Capecitabine compared with Bevacizumab plus Capecitabine in patients treated with 
Bindu S M et.al. Eribulin mesylate in treated metastatic breast cancer patients: a retrospective study of tumor response and adverse effects

metastatic breast cancer. JCO Vol23; February 2005; 164-69

17. Blum JL, Barrios $\mathrm{CH}$. Feldman $\mathrm{N}$ et al. Pooled analysis of individual patient data from capecitabine monotherapy clinical trials in locally advanced or metastatic breast cancer; Breast Cancer Res Treat. 2012;136:777-88

18. Colomer R, Llombert, CussacA,Lluch A et al ; Biweekly Paclitaxel plus gemcitabine in advanced breast cancer Phase II trial and predictive value of Her2R extracellular domain; Ann Oncol 2004 Feb; 15(2) 201-6

19. Polethi P, Ghilardi V, Livraghi L, Milesi L, Rota Caremoli E. et al. Eribulin mesylate in heavily pretreated metastatic breast cancer patients: Current practice in an Italian community hospital. Future Oncol. 2014; 10:233-9
20. AthinaStravodimou, Khalil Zaman. Ioannis et al: Vinorelbine with or without Trastuzumab in Metastatic Breast Cancer-A retrospective single institute Series: Internal Scholarly Research Notices/ Vol: 2014; ArticleID289836/https://doiorg/10. $1155 / 2014 / 289836$

21. Garrrone O, Montemurro F, Saggia $C$ et al. Eribulin in pretreated metastatic breast cancer patients; results of the TROTTER trial; Multicentre retrospective study of eribulin in real life, Sprinerto 2016;5:59

How to cite this article: Bindu S M, P L Rema, Ninan PJ. Eribulin Mesylate in Treated Metastatic Breast Cancer Patients: A Retrospective Study of Tumor Response and Adverse Effects. Gal Int J Health Sci Res. 2021; 6(2): 37-44. DOI: https://doi.org/10.52403/ gijhsr.20210405 